\title{
Exponentially Growing Tearing Modes in Rijnhuizen Tokamak Project Plasmas
}

\author{
F. Salzedas, * F. C. Schüller, A. A. M. Oomens, and the RTP Team \\ FOM-Instituut voor Plasmafysica "Rijnhuizen," Association Euratom-FOM, Trilateral Euregio Cluster, \\ P.O. Box 1207, 3430 BE Nieuwegein, The Netherlands
}

(Received 14 March 2001; published 5 February 2002)

\begin{abstract}
The local measurement of the island width $w$, around the resonant surface, allowed a direct test of the extended Rutherford model [P. H. Rutherford, PPPL Report-2277 (1985)], describing the evolution of radiation-induced tearing modes prior to disruptions of tokamak plasmas. It is found that this model accounts very well for the observed exponential growth and supports radiation losses as being the main driving mechanism. The model implies that the effective perpendicular electron heat conductivity in the island is smaller than the global one. Comparison of the local measurements of $w$ with the magnetic perturbed field $\tilde{B}$ showed that $w \propto \tilde{B}^{1 / 2}$ was valid for widths up to $18 \%$ of the minor radius.
\end{abstract}

DOI: 10.1103/PhysRevLett.88.075002

The identification of the main driving mechanism of the observed MHD (magnetohydrodynamics) instabilities in a tokamak plasma is of considerable interest. Such knowledge of the nature of the instability can be very helpful in the choice of a stabilization procedure needed to avoid degradation of confinement or plasma disruption. Disruptions are the most dangerous instabilities in tokamak plasmas, and the precursor of density limit disruptions is often dominated by an $m / n=2 / 1$ MHD instability [1] (where $m$ and $n$ are the poloidal and toroidal Fourier mode numbers, respectively). In this Letter, we study the exponential increase of an $m / n=2 / 1$ tearing mode observed prior to a density limit disruption [see Fig. 1(a)] in RTP (Rijnhuizen Tokamak Project, circular limiter, minor radius $a=0.16 \mathrm{~m}$ and major radius $R_{0}=0.72 \mathrm{~m}$ ). Similar exponential growth is observed also in other tokamaks [2]. Comparison with the extended Rutherford [3] model supports radiative energy losses as the main driving mechanism for this tearing mode. To avoid confusion with the well-known neoclassical tearing modes, we, henceforward denominate these instabilities as radiative induced tearing mode or RTM. The study of RTM is also very important because future large tokamaks such as ITER are expected to work close to $100 \%$ edge radiation to reduce thermal load on the divertor.

In the plasmas studied here, the plasma current $I_{P}=$ $100 \mathrm{kA}$, the safety factor $q_{a} \approx 4$, the ratio of the plasma energy to the magnetic field energy, $\beta$, was low, and at the time the RTM is observed collisionality is high. So neoclassical effects are disregarded. The filling gas was $\mathrm{He}$ and the density was ramped up using $\mathrm{Ne}$ gas injection. Both noble gases are not absorbed by the vessel wall. This allowed a better and reproducible control of the electron density than with hydrogenic plasmas. Moreover, the density at disruption could be kept below the cutoff density of the electron cyclotron emission (ECE) heterodyne radiometer, used to measure the time evolution of the electron temperature at 20 radial positions along a horizontal chord. Electron temperature and density were measured at three time points with a high spatial resolution Thomson
PACS numbers: 52.55.Tn, 52.35.Py

scattering (TS) system. Sawteeth are always observed in these discharges [Fig. 1(b)].

One of the difficulties in the analysis of the behavior of tearing modes is to relate the measured perturbed poloidal magnetic field $\tilde{B}_{\theta}\left(r_{c}\right)$ [Fig. 1(a)], where $r_{c}$ is the radius of the pickup coils, with the unknown perturbed radial magnetic field $\tilde{B}_{r}\left(r_{s}\right)$ at the mode resonant surface, $r_{s}$, that is the quantity whose dynamics is predicted by theory [4]. The usual procedure consists $[5,6]$ of approximating the toroidal plasma by a cylindrical one and then to use the
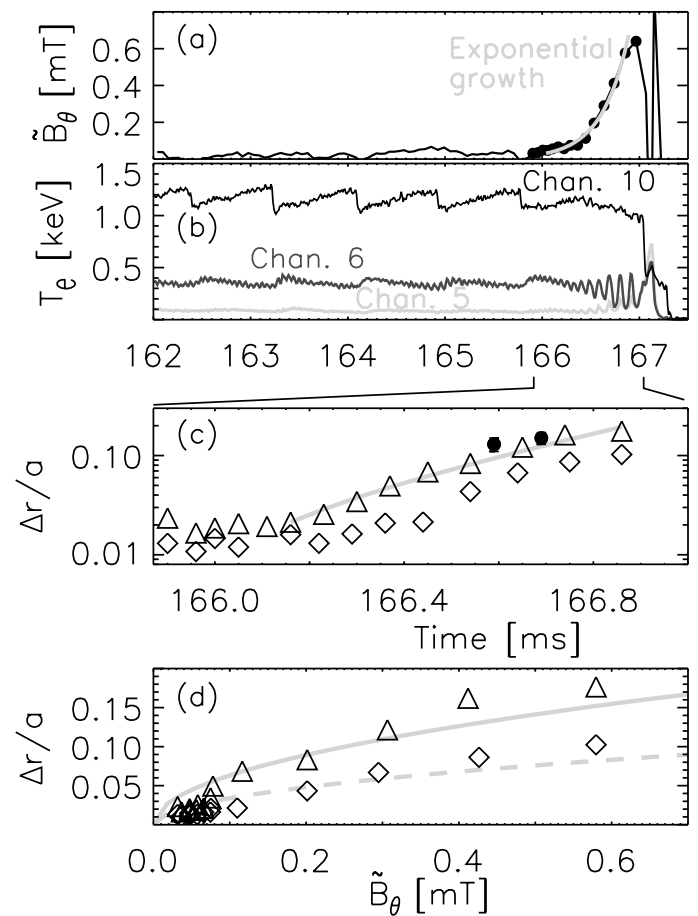

FIG. 1. (a) Amplitude of $\tilde{B}_{\theta}$. (b) ECE $T_{e}$ in the center (channel 10) and in the vicinity of the $q=2$ surface (channels 6 and 5) [see Fig. 2(a)]. (c) $\Delta r / a$ calculated with (4). The solid circles indicate the island width estimated from TS [see Figs. 2(c)-2(e)]. The gray line is the fit to Eq. (10). (d) $\Delta r / a$ plotted against $\tilde{B}_{\theta}$. The dashed (solid) gray line represents Eq. (6) [Eq. (5)]. Symbol $\triangle(\diamond)$ refers to LFS (HFS). 
current profile as found from one of the methods described, for example, in [7], to solve the equation

$$
\frac{d^{2} \psi}{d r^{2}}+\frac{1}{r} \frac{d \psi}{d r}-\left(\frac{m^{2}}{r}+\frac{\mu_{0} \frac{d j_{z 0}}{d r}}{B_{\theta 0}\left(1-\frac{n}{m} q\right)}\right) \psi=0,
$$

using $\tilde{B}_{\theta}\left(r_{c}\right)$ as a boundary condition, to find $\tilde{B}_{r}\left(r_{s}\right)$, where $\psi$ is a scalar potential that is related to $\tilde{\mathbf{B}}$ by

$$
\tilde{\mathbf{B}}=\nabla \psi \times \hat{z}=-\frac{1}{r} \frac{\partial \psi}{\partial \theta} \hat{r}+\frac{\partial \psi}{\partial r} \hat{\theta} .
$$

The $z$ component of the equilibrium plasma current is $j_{z 0}$, and $B_{\theta 0}$ is the poloidal component of the equilibrium magnetic field.

In the case that the plasma current outside the resonant surface can be neglected and the wall acts as a perfect conductor, Eq. (1) has an explicit solution. Then, using (2), the $m$ component of $\tilde{B}_{\theta}$ at $r_{c}$ is related to the $m$ component of $\tilde{B}_{r}$ at $r_{s}$ by

$$
\tilde{B}_{\theta m}\left(r_{c}\right)=\tilde{B}_{r m}\left(r_{s}\right)\left(\frac{r_{s}}{r_{c}}\right)^{(m+1)} \frac{1+\left(\frac{r_{c}}{r_{w}}\right)^{2 m}}{1-\left(\frac{r_{s}}{r_{w}}\right)^{2 m}},
$$

where $r_{w}$ is the radius of the vessel wall.

In the methods which use (1) and (3) with measurements of external pickup coils, the mode evolution is described via the amplitude of the perturbed magnetic field. In what follows, another method to measure the spatial amplitude of the perturbations, directly and locally around the resonant surface, will be used. Basically, it relies on the assumption that close to and outside of the separatrix of a tearing mode the electron temperature is a flux function [8]. In this case, a change $\Delta w$ of the island width will cause a proportional displacement $\Delta r$ of the flux surfaces in the neighborhood of the separatrix. The displacement $\Delta r$ is given by

$$
\Delta r=\frac{\Delta T}{\frac{d T_{0}}{d r}},
$$

where $T_{0}$ is the temperature profile, measured radially along the $X$ point and $\Delta T$ is the difference in temperature between the two points at the two angles that correspond with the $X$ point and the $O$ point [9]. It is also implicitly assumed that the plasma is incompressible. Figure 1(c) shows $\Delta r / a$, derived both from the low field side (LFS), channel 6 of the radiometer, and on the high field side (HFS), channel 16. The position of these channels is shown in Fig. 2(a) and a pictorial illustration of $\Delta r$ (LFS) is shown in Fig. 2(f). It is expected that the smaller values of $\Delta r$ have lower accuracy since the island separatrix
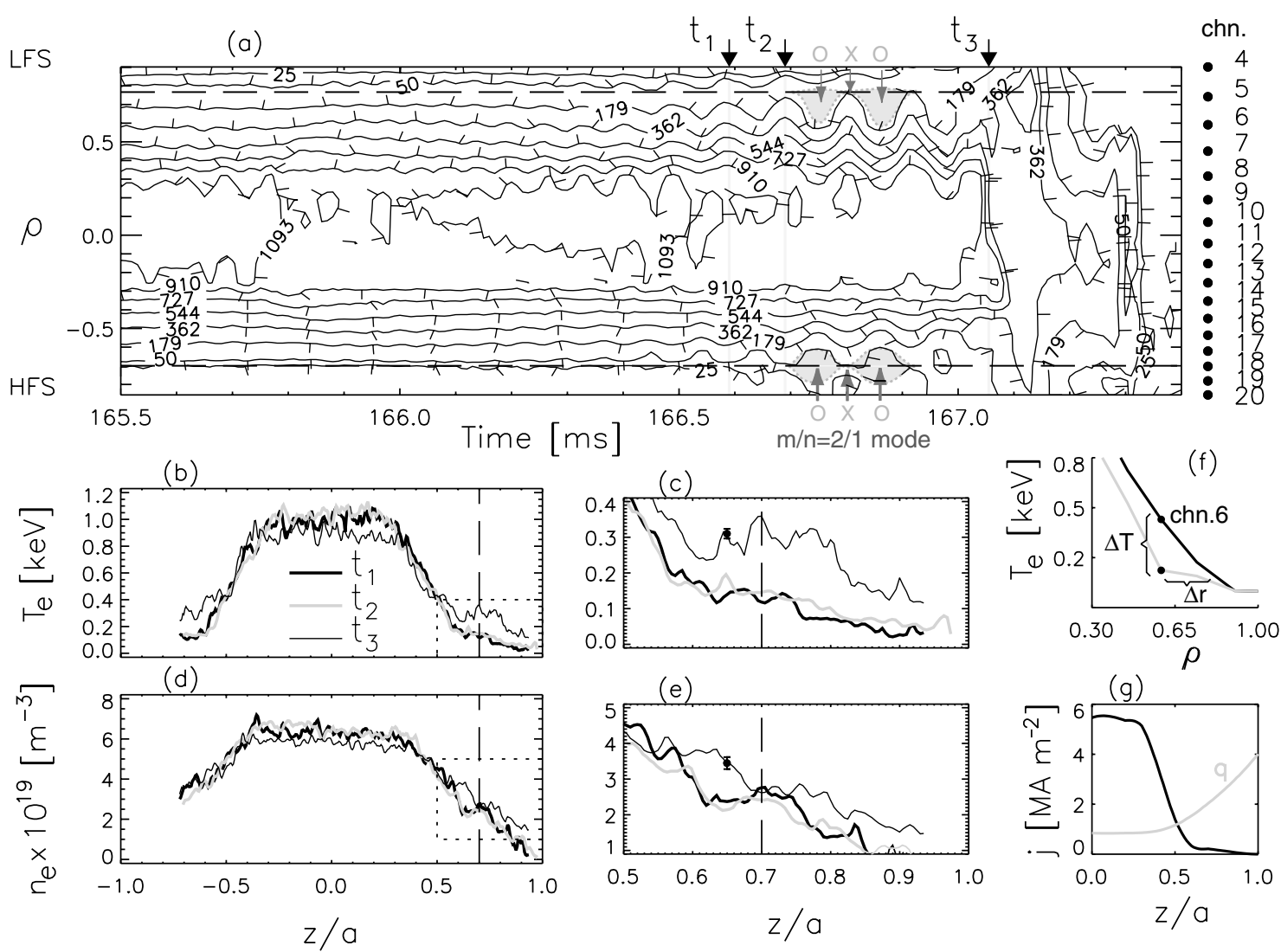

FIG. 2. (a) ECE $T_{e}(r, t)$ isotherms. The channels position is indicated at the right. A sketch of the $m=2$ mode separatrix is illustrated by the dotted lines around the gray areas. (b) TS $T_{e}$ profiles at $t_{1}, t_{2}$, and $t_{3}$. (c) Zoom in on (b). For clarity, only one typical error bar is shown. (d) and (e) are the same as before for $n_{e}(r)$. The dashed line indicates the calculated radial position of the $q=2$ surface. (f) Illustration of (4). Gray (black) line, ECE $T_{e}(r)$ passing through the $O(X)$ point. $(\mathrm{g}) j(r)$ and $q(r)$ calculated from $T_{e}\left(t_{1}\right)$. 
is further away from the ECE channels. On the other extreme, if the separatrix crosses the ECE channel then the definition (4) is not applicable.

A characteristic of $m=2$ islands in tokamaks is visible in the ECE measurements, as shown in Fig. 2(a), namely that the deformation of isotherms is symmetric in relation to the resonant surface on the HFS [see illustration in Fig. 2(a)]. On the LFS it is more difficult to localize the $X$ point, since the island grows asymmetrically in respect to the resonant surface, i.e., much more towards the center of the plasma than to the edge. In this case it is expected that on the LFS the strongly asymmetric island leads to $\Delta r \simeq w$, while on the HFS a symmetric island gives $\Delta r \simeq w / 2$. Figure 1(c) confirms this expectation with $\Delta r(\mathrm{LFS}) \simeq 2 \Delta r$ (HFS). Moreover, the estimated island width from the TS [10], measured along a vertical chord, comes very close to the values of $\Delta r$ measured on the LFS [see Figs. 1(c) and 2(c)-2(e)].

In Fig. 1 (d) $\Delta r$ is plotted against $\tilde{B}_{\theta}\left(r_{c}\right)$, for values up to $18 \%$ of the minor radius, which are measured just $100 \mu \mathrm{s}$ before the onset of the disruption. Without any assumption on the plasma equilibrium, the best fits to the experimental data give the following for the LFS and HFS:

$$
\begin{aligned}
& \Delta r(\mathrm{LFS})=(1.04 \pm 0.08) \sqrt{\tilde{B}_{\theta}\left(r_{c}\right)}, \\
& \Delta r(\mathrm{HFS})=(0.56 \pm 0.05) \sqrt{\tilde{B}_{\theta}\left(r_{c}\right)} .
\end{aligned}
$$

We can compare the experimental relation (5) with the well-known expression for the island width that relates the spatial and magnetic mode amplitudes:

$$
w=4 \sqrt{\frac{r_{s} q}{m B_{\theta} q^{\prime}}} \sqrt{\tilde{B}_{r}\left(r_{s}\right)} .
$$

Because of the radiative contraction of the current profile, the current for $r>r_{s}$ can be neglected so we can use (3) to obtain $\tilde{B}_{r}$ for $m=2$. Using the values from Table I that were calculated from TS $T_{e}\left(t_{1}\right)$ [see Figs. 2(b) and $2(\mathrm{~g})]$, the following is obtained:

$$
w=(1.14 \pm 0.25) \sqrt{\tilde{B}_{\theta}\left(r_{c}\right)} .
$$

The agreement between (8) and the experimentally derived (5) is very good. This supports the calculation of $w$ in the cylindrical approximation used until now.

Experimental evidence indicates that the equilibrium parameters, $B_{\theta}\left(r_{s}\right), r_{s}$, and $q^{\prime}\left(r_{s}\right)$, do not change noticeably in time during the island exponential growth. For $r_{s}$ this can be seen unambiguously at the HFS in Fig. 2(a). Relatively to $B_{\theta}\left(r_{s}\right)$ and $q^{\prime}\left(r_{s}\right)$, since the current diffusion time is typically $10-20 \mathrm{~ms}$, it is very unlikely that these

TABLE I. Equilibrium parameters at $r=r_{s}$ calculated from $T_{e}\left(t_{1}\right)$ in Fig. 2(b), assuming $j_{z} \propto T_{e}\left(t_{1}\right)^{3 / 2}$.

\begin{tabular}{cccccc}
\hline \hline $\begin{array}{c}r_{s} \\
(\mathrm{~m})\end{array}$ & $\begin{array}{c}T_{e} \\
(\mathrm{eV})\end{array}$ & $\begin{array}{c}j \\
\left(\mathrm{MA} \mathrm{m}^{-2}\right)\end{array}$ & $\begin{array}{c}q^{\prime} \\
\left(\mathrm{m}^{-1}\right)\end{array}$ & $\begin{array}{c}B_{\theta} \\
(\mathrm{T})\end{array}$ & $\begin{array}{c}\eta \\
\left(10^{-6} \Omega \mathrm{m}\right)\end{array}$ \\
\hline 0.113 & 127 & 0.211 & 32.7 & 0.165 & 4.12 \\
\hline \hline
\end{tabular}

parameters change significantly during the $0.6 \mathrm{~ms}$ of the mode exponential growth. So, assuming that during this period the equilibrium parameters do not show noticeable changes, Fig. 1(d) provides a direct demonstration that (7) is still valid up to island sizes of the order of $18 \%$ of the minor radius. This feature should be emphasized because the use in the literature (e.g., [5,6]) of (7) for island sizes of this magnitude is based only on the magnetic measurements performed outside the plasma, which are extrapolated inwards up to $r_{s}$.

Before the exponential growth starts, it is observed that, on average, the amplitude of $\tilde{B}_{\theta}$ grows algebraically for a period of $\approx 20 \mathrm{~ms}$. During this period [of which only the last $4 \mathrm{~ms}$ are shown in Fig. 1(a)], the growth of the mode is not monotonic but shows an irregular modulation with the amplitude now and then decreasing practically to zero. This modulation is often correlated with a sawtooth crash. This complex behavior will not be discussed here. Instead we will analyze only the exponential phase using the extended Rutherford model [3],

$$
\frac{d w}{d t}=C_{1} \frac{\eta}{\mu_{0}} \Delta^{\prime}-C_{2} \frac{\tilde{P}_{T}}{\chi_{\perp \text { eff }}^{\text {island }}\left\langle T_{e}\right\rangle} w,
$$

where $\eta$ is the plasma resistivity, $C_{1}=1.22$, and $C_{2}=$ $0.9 \eta j_{z} q /\left(B_{\theta} q^{\prime}\right)$ with all the quantities taken at the resonant surface. $\tilde{P}_{T}$ is the total power density per particle in the island and the mode is destabilized if $\tilde{P}_{T}$ is negative. $\chi_{\perp \text { eff }}^{\text {island }}$ and $\left\langle T_{e}\right\rangle$ are the effective perpendicular electron thermal conductivity and the average electron temperature in the island, respectively.

From 165.8 to $166.2 \mathrm{~ms}$, the evolution of the amplitude of the mode magnetic field could be well fitted with a quadratic increase with time implying a linear increase in island width. During this period, losses by radiation inside the island are still not important, and so the second term of (9) is negligible. The value of $\Delta^{\prime}$ can then be estimated from the linear increase of the island width. Using the value of $\eta$ from Table I, we obtain $\Delta^{\prime}=2$.

Between 166.2 and $166.8 \mathrm{~ms}$, the increase in the mode amplitude is very well described by an exponential fit. This can be explained by a sudden onset of the second term of (9) with $\tilde{P}_{T}<0$. An abrupt negative value of $\tilde{P}_{T}$ is perfectly possible due to the nature of radiative power losses. This is an effect of the inverse proportionality between radiated power density and the electron temperature. If impurities have been accumulating inside the island, once the temperature on the $O$ point is low enough such that radiative recombination of these impurities can occur, a small decrease of temperature will lead to an increase in radiation loss. Energy in the island is then dissipated faster, setting abruptly $\tilde{P}_{T}<0$. The heat flowing into the island can be enough to keep $\tilde{P}_{T}$ constant, during the exponential growth, as both ECE and TS indicate.

The high spatial resolution temperature and density profiles inside the island are neither flat nor monotonic, but irregular [Fig. 2(c)]. On both $t_{1}$ and $t_{2}$ profiles, despite the 
maxima and minima, in the island the average value of $T_{e}$ does not change. This indicates that the heat flow inside the island is not purely diffusive. The ECE [Fig. 2(a)], which has lower spatial resolution than TS and so does not detect the irregularity found by the TS profiles, also shows the island growing without significant changes in $\left\langle T_{e}\right\rangle$. Secondary maxima in $T_{e}$ and $n_{e}$ were also observed in TEXTOR $[11,12]$.

From the data of Fig. 1(c), it is possible to estimate the ratio of $\tilde{P}_{T} / \chi_{\perp \text { eff }}^{\text {island }}$. Assuming that $\tilde{P}_{T}$ is constant during the short time interval in which the mode grows exponentially, integration of (9) gives

$$
w(t)=\frac{a_{1}}{a_{2}}+\left(w_{0}-\frac{a_{1}}{a_{2}}\right) e^{-a_{2} t},
$$

where $w_{0}=w(166.2)=0.02 a, a_{1}=c_{1} \frac{\eta \Delta^{\prime}}{\mu_{0}}=8$, and

$$
a_{2}=\frac{0.9 \eta j_{z} q}{B_{\theta} q^{\prime}\left\langle T_{e}\right\rangle} \frac{\tilde{P}_{T}}{\chi_{\perp \mathrm{eff}}} .
$$

The fitting of (10) to the data of Fig. 1(c) (dashed line) gives $a_{2}=-2.3 \times 10^{3} \mathrm{~s}^{-1}$. From (11) and Table $\mathrm{I}$ it is found

$$
\tilde{P}_{T} / \chi_{\perp \text { eff }}^{\text {island }}=-1.0 \times 10^{6} \mathrm{eV} \mathrm{m}^{-2} .
$$

Both $\tilde{P}_{T}$ and $\chi_{\perp \text { eff }}^{\text {island }}$ are unknown, but it is possible to estimate its range of values. At the time the TS $T_{e}\left(t_{1}\right)$ profile is measured, the energy confinement time is $\tau_{e}=3.4 \mathrm{~ms}$ which gives a global effective perpendicular electron thermal conductivity $\chi_{\perp \text { eff }}^{\text {global }}=a^{2} /\left(4 \tau_{e}\right)=$ $1.9 \mathrm{~m}^{2} \mathrm{~s}^{-1}$. If $\chi_{\perp \text { eff }}^{\text {island }}$ was the same as $\chi_{\perp \text { eff }}^{\text {global }}$, then $\tilde{P}_{T}=-1.9 \times 10^{5} \mathrm{eV} \mathrm{s}^{-1}$ per particle which means that during the $0.6 \mathrm{~ms}$ of exponential growth an average of $1140 \mathrm{eV}$ per particle would have to be dissipated in the island. Such a value is very large since during the same period the temperature in channel 6 decreases $215 \mathrm{eV}$, while in channel 5 it increases only $65 \mathrm{eV}$ [Fig. 1(b)]. The difference of $150 \mathrm{eV}$ between these two channels is almost 1 order of magnitude smaller than the previously found value $1140 \mathrm{eV}$. Assuming that only $150 \mathrm{eV}$ were dissipated in the island, then $\tilde{P}_{T} \approx-2.5 \times 10^{5} \mathrm{eV} \mathrm{s}^{-1}$ per particle (which corresponds to $\approx-70 \mathrm{~kW}$ ). This value is in good agreement with the radiation losses in the outer plasma layers at comparable temperature values and is very close to the minimum electron cyclotron power of $90 \mathrm{~kW}$ that was found necessary to stabilize the $m=2$
RTM in similar discharges $[9,13])$. So from (12) it follows that $\chi_{\perp \text { eff }}^{\text {island }} \approx 0.25 \mathrm{~m}^{2} \mathrm{~s}^{-1}$.

This value of the effective electron heat conductivity inside the island is almost 1 order of magnitude smaller than the global effective electron heat conductivity of the plasma. This reduction in diffusivity inside the island is in agreement with previous indications $[11,12,14]$. Moreover, it is corroborated by the observation in $\operatorname{TS} T_{e}\left(t_{1}, t_{2}\right)$ of the secondary maxima in temperature and density inside the island, relatively to the separatrix. The $T_{e}$ maxima are more pronounced in TS $T_{e}\left(t_{3}\right)$. Despite this profile was measured immediately after the exponential growth $\tilde{B}_{\theta}\left(r_{c}\right)$ does not indicate any strong change in the island structure. Only later at $t=167.2 \mathrm{~ms}$, during the disruption $\tilde{B}_{\theta}\left(r_{c}\right)$ shows a sharp spike, indicating the probable destruction of the island.

One of the authors (F. Salzedas), was supported by the Portuguese Foundation for Science and Technology FCT under Programa PRAXIS XXI-Grant No. BD/4531/94. This work was performed under the Euratom-FOM Association agreement, with financial support from the Dutch research organization NWO and Euratom.

* Present address: Centro de Fusão Nuclear, Association Euratom-IST, Av. Rovisco Pais, 1049-001 Lisboa, Portugal.

Electronic address: fsal@cfn.ist.utl.pt

[1] F. C. Schüller, Plasma Phys. Controlled Fusion 37, A135 (1995).

[2] M. Schittenhelm et al., Nucl. Fusion 37, 1255 (1997).

[3] P. H. Rutherford, PPPL Report-2277 (1985).

[4] P. H. Rutherford, Phys. Fluids 16, 1903 (1973).

[5] Z. Chang et al., Phys. Rev. Lett. 74, 4663 (1995).

[6] B. Carreras et al., Nucl. Fusion 19, 1423 (1979).

[7] H. Soltwisch, Plasma Phys. Controlled Fusion 34, 1669 (1992).

[8] R. Fitzpatrick, Phys. Plasmas 2, 825 (1995).

[9] F. Salzedas, Ph.D. thesis, Universiteit Utrecht, 2000 (URL: http://www.library.uu.nl/digiarchief/dip/diss/1940669/inhoud.htm).

[10] TS has a very high spatial resolution but only one point in time is given while ECE has a high temporal but low spatial resolution. Both effects cause some error in the location of the separatrix.

[11] P. C. de Vries et al., Plasma Phys. Controlled Fusion 39, 439 (1997).

[12] P. C. de Vries et al., Nucl. Fusion 37, 1641 (1997).

[13] F. Salzedas et al. (to be published).

[14] B. P. van Milligen et al., Nucl. Fusion 33, 1119 (1993). 